\title{
Matriz de acetato como opción para reconstrucción de coronas y restitución de la guía anterior en dentición primaria
}

\author{
Acetate matrix as an option for dental crowns reconstruction \\ and earlier guide restoration in primary dentition
}

\author{
Zendy J. Sosa Vilca' \\ Universidad Continental \\ zeja40@hotmail.com
}

\author{
Mario C. Elías Podestá2 \\ Universidad Continental
}

\author{
Jesús M. Cóndor Astucuri ${ }^{3}$ \\ Universidad Continental
}

\section{RESUMEN}

Los objetivos de este estudio han sido restituir la guía anterior de la dentición primaria, mediante el uso de la matriz de acetato que es una de las alternativas de toda esta gama para la reconstrucción; alinear los dientes de acuerdo al biotipo facial, logrando una estética apropiada y permitir también una correcta fonación; devolver una de las funciones de los dientes anteriores que es la de corte y así favorecer la correcta masticación; lograr recuperar la autoestima, pues el niño era objeto de burlas por parte de sus compañeros de clases y amigos del vecindario. El paciente fue de sexo masculino, de 5 años y 3 meses de edad que acudió a la Clínica de la Segunda Especialidad de Odontopediatría de la Universidad Continental en Huancayo, éste presentaba lesiones cariosas amplias en el sector anterosuperior; al elaborar la Historia Clínica, la madre informa que el paciente padece de Asma desde la edad de 12 meses. En la anamnesis relató que la alimentación fue lactancia mixta y amamantamiento nocturno con ausencia de limpieza bucal; el estudio radiográfico confirmó lo observado clínicamente: caries amplias mesiales y distales a nivel de la corona. Al finalizar la intervención se puede mencionar que los resultados fueron óptimos en la parte clínica, estética y funcional. La matriz de acetato es una alternativa óptima para la confección de las coronas de resina y así se reestableció la guía anterior, masticación, fonación, estética, logrando recuperar el autoestima y la confianza perdida en sí mismo debido a las burlas en su entorno escolar, consiguiendo un cambio positivo en su personalidad.

\section{ABSTRACT}

The objectives were to restore the earlier guide of primary dentition, by using the acetate matrix which is one of the alternatives of all this range for reconstruction; align teeth according to the facial biotype, achieving an appropriate aesthetic and also allow a proper phonation; return one of the earlier teeth functions that is the cutting and thus promote the proper chewing; achieve to recover self-esteem, because the child was tease by their classmates and neighborhood friends. The patient was male, 5 years old and 3 months old who came to the Pediatric Dentistry Second Specialty Clinic from the "Universidad Continental" in Huancayo, he had large carious lesions in the anterosuperior sector; when we made the clinical history, the mother reported that the patient suffers from asthma since he was 12 months old. In the anamnesis the food was mixed breastfeeding and nocturnal breastfeeding with no teeth cleaning; the radiographic study confirmed the clinically observation: mesial and distal large caries in the crown level. After the intervention optimal results can be noted in the clinical, cosmetic and functional part. The acetate matrix is an excellent alternative for making resin crowns so the earlier guide, chewing, phonation and aesthetics were restored, also achieving to recover self-esteem and the lost confidence because of the teasing in their school environment, getting a positive change in his personality.

Keywords: Pulpectomies, acetate matrix, oral rehabilitation.

Palabras clave: Pulpectomías, matriz de acetato, rehabilitación oral.

Historial del artículo:

Recibido: 14 de marzo de 2015. Aprobado: 3 de mayo de 2015. Disponible en línea: 30 de junio de 2015

1 Cirujano Dentista Residente del Primer año de la Segunda Especialidad de Odontopediatría de la Universidad Continental

2 Ph.D, Especialista, Cirujano Dentista, Director de la Segunda Especialidad de Odontopediatría de la Universidad Continental.

3 Especialista, Cirujano Dentista, Docente del curso de Odontopediatría de la Universidad Continental. 


\section{INTRODUCCIÓN}

La caries dentaria es una dolencia que afecta las estructuras mineralizadas de los dientes (esmalte $y$ dentina) y el cemento. Es crónico y, generalmente, cuando no es tratada, progresa hasta la destrucción total de la pieza dentaria (1).

Cuando los niños pierden precozmente sus dientes primarios, sea por trauma o por caries, necesitamos rehabilitarlos estética y funcionalmente hasta que ocurra la aparición de los dientes sucesores permanentes (2), una de las alternativas es la confección de coronas de resina con matriz de acetato hechas a partir de un stock de dientes anteriores de acrílico.

Las consecuencias de la destrucción de las estructuras del esmalte, dentina hasta llegar a la pulpa dental o los dientes anteriores que es la de corte, favoreciendo la masticación y así mejorar el proceso de digestión; lograr recuperar la autoestima, pues el niño era objeto de burlas por parte de sus compañeros de clases y amigos del vecindario, creando en él una personalidad introvertida e insegura debido al aspecto que presentaba cuando sonreía, según refiere la madre.

\section{Reporte de caso}

Paciente de sexo masculino de 5 años y 3 meses de edad, acude a la Clínica de la Segunda Especialidad de Odontopediatría de la Universidad Continental, presentando lesiones cariosas amplias en el sector anterosuperior (Figura № 1), siendo objeto de burlas por parte de sus compañeros de clases, logrando la pérdida de la autoestima del niño y generando

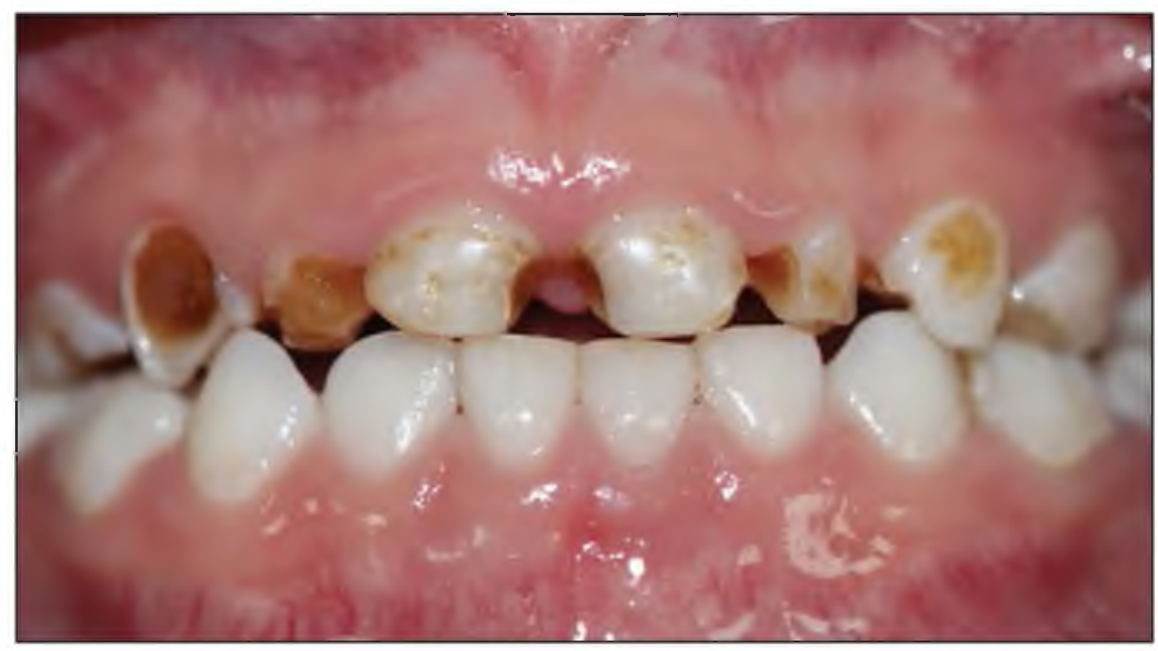

Figura $N^{\circ} 1$ : Procesos cariosos con destrucción amplia.

destrucción total de ella son variadas, inflamación, dolor, infección, produciendo abcesos con o sin fístulas - llegando inclusive a producir celulitis que a veces nos demanda hasta la hospitalización.

Para rehabilitar los dientes anteriores podemos utilizar coronas de resina acrílica confeccionados en el laboratorio (2).

En este artículo se narra la restauración del sector anterior con coronas de resina confeccionadas con matriz de acetato como una alternativa para la rehabilitación. Se optó por elegir dicha técnica con los objetivos de restituir la guía anterior, perdida debido a la destrucción de las coronas por caries amplias, en la dentición primaria; alinear los dientes de acuerdo al biotipo facial que presenta el paciente, logrando de esta manera una estética adecuada y ayudando a la correcta pronunciación de las palabras, que es muy importante para la interrelación con su medio escolar y familiar; devolver una de las funciones de malestar en el entorno familiar.

Al elaborar la Historia Clínica, la madre informa que el paciente padece de Asma desde la edad de 12 meses. En la anamnesis relató que la alimentación fue lactancia mixta y amamantamiento nocturno con ausencia de limpieza bucal.

El estudio radiográfico confirmó lo observado clínicamente: caries amplias mesiales y distales a nivel de la corona (Figura №2). Después del análisis radiográfico se elaboró el plan de tratamiento.

Se inicia con la orientación a la madre sobre la higiene (cepillado, uso de hilo dental y uso de enjuague) y al niño, con ayuda de cartilla motivadora elaborada con ejemplos prácticos para el entendimiento propio de la edad.

Se continúa el tratamiento por cuadrantes, con la finalidad de tener mejor resultado y mayor rapidez. La 
anestesia y el dique de goma siempre fueron utilizados. La remoción de la caries fue el siguiente paso, previa aplicación de anestesia y aislamiento absoluto, las pulpectomías se realizaron en las piezas dentarias 51 , $52,53,61,62$ y 63; y la obturación correspondiente de los conductos radiculares con óxido de zinc $y$ eugenol (Figuras $\mathrm{N}^{\circ} 3$ y 4 ).

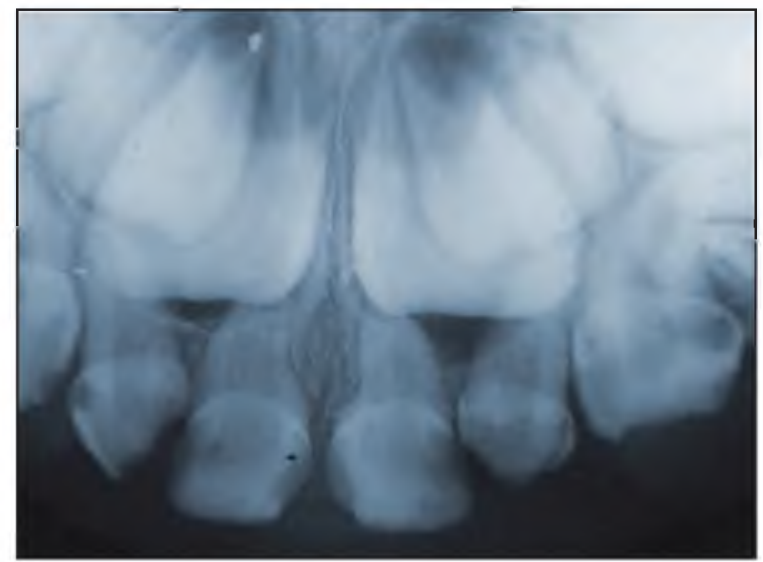

Figura № 2: Radiografía de diagnóstico.

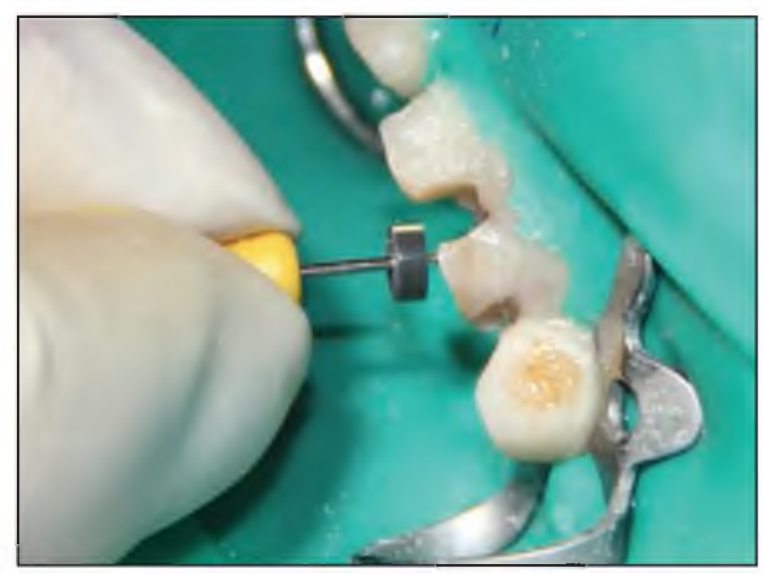

Figura $\mathrm{N}^{\circ} 3$ : Exéresis pulpar e instrumentación del conducto.

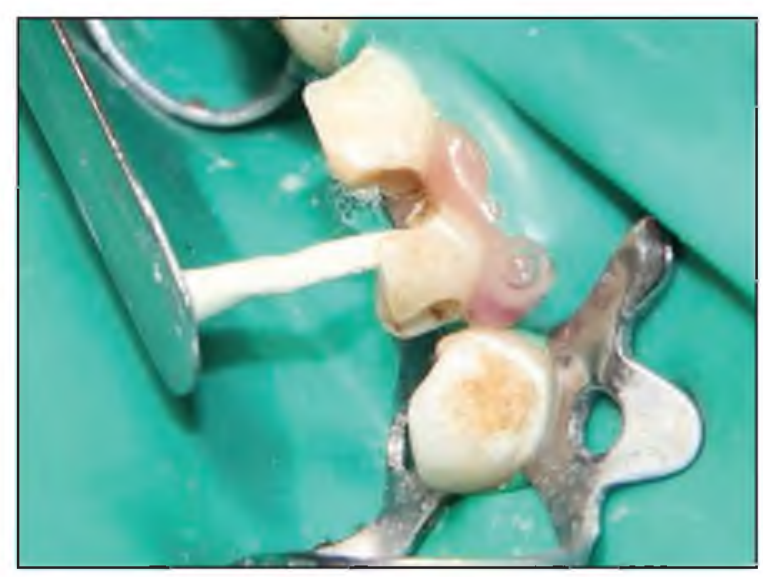

Figura $\mathrm{N}^{\circ} 4$ : Obturación del conducto.
Después de realizar la obturación de los conductos (Figura $N^{\circ}$ 5), se procede a la colocación del espigo muñón confeccionado de acrílico en la pieza dentaria 52, debido a que presentaba una destrucción mayor a los tres cuartos de la porción coronaria que las demás piezas, y por último se coloca una base de ionómero de vidrio antes de la preparación de las coronas con la matriz de acetato.

Las coronas fueron preparadas en dos citas debido a lo minucioso del procedimiento y la condición cooperativa del niño.

El aislamiento absoluto del campo operatorio es importante también en esta fase para el control de la humedad y el éxito del tratamiento.

Después de la elección del color de la resina, se acondicionó las piezas dentarias con ácido fosfórico al $37 \%$ por $15^{\prime}$. Se procede al lavado con agua en abundancia por 30'.

Inmediatamente después del secado, se aplica el sistema adhesivo Single Bond ( $3 M$ ESPE) con micropincel y se fotopolimeriza por el tiempo de 20', quedando la pieza dentaria lista para la recepción de la resina.

Se selecciona del stock de las piezas dentarias de acrílico para la confección de la matriz de acetato el apropiado que cumple los requisitos de la anatomía dental y la distancia mesio distal del espacio que se requiere (Figura $\mathrm{N}^{\circ}$ 6); obtenida la matriz de acetato apropiada, se adapta a la pieza dentaria siguiendo los contornos anatómicos de sus caras vestibular, palatino, mesial y distal (Figura $N^{\circ} 7$ ), recortando los excesos cervicales con una tijera curva. Una pequeña perforación a nivel incisal se realizó para facilitar el derrame del exceso del material.

Después de rellenar la resina seleccionada al interior de la matriz de acetato y luego trasladarlo a la pieza dentaria ya preparada para la corona, se remueve los excesos expulsados por el orificio y a nivel cervical, luego se fotopolimeriza por todas las caras de la restauración.

La matríz de acetato para ser retirada requiere de un corte con hoja de bisturí de cervical a incisal y luego es removido con un explorador (Figura $\mathrm{N}^{\circ} 8$ ).

Finalmente se retira el aislamiento absoluto; se realiza el ajuste oclusal verificando que no existan interferencias en estática y en dinámica mandibular con el auxilio del papel de articular (Figura $\mathrm{N}^{\circ}$ 9). 


\section{DISCUSIÓN}

Frecuentemente, encontramos problemas en dientes anteriores de niños, involucrando estética y función. Esos problemas son derivados de traumatismos con consecuencias de alteraciones cromáticas en función de hemorragia (diente vital) o necrosis pulpar, de mal formaciones del tipo hipoplasia o hipocalcificación, y

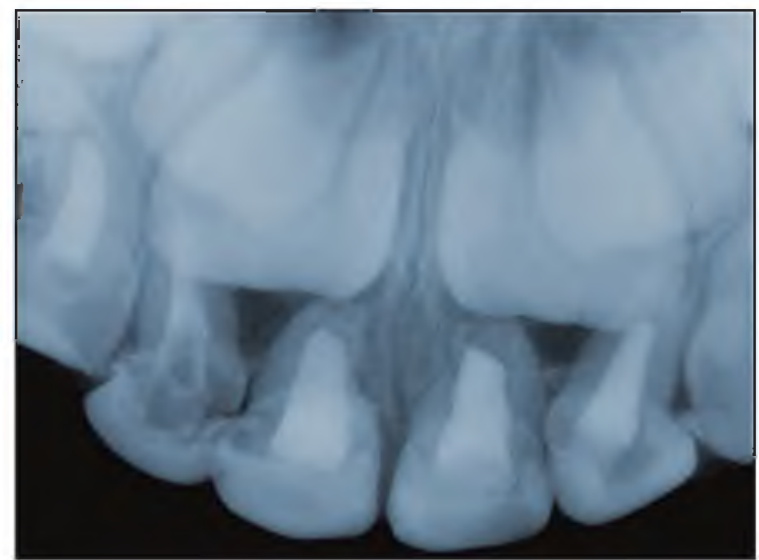

Figura $N^{0} 5$ : Vista radiográfica de conductos obturados.

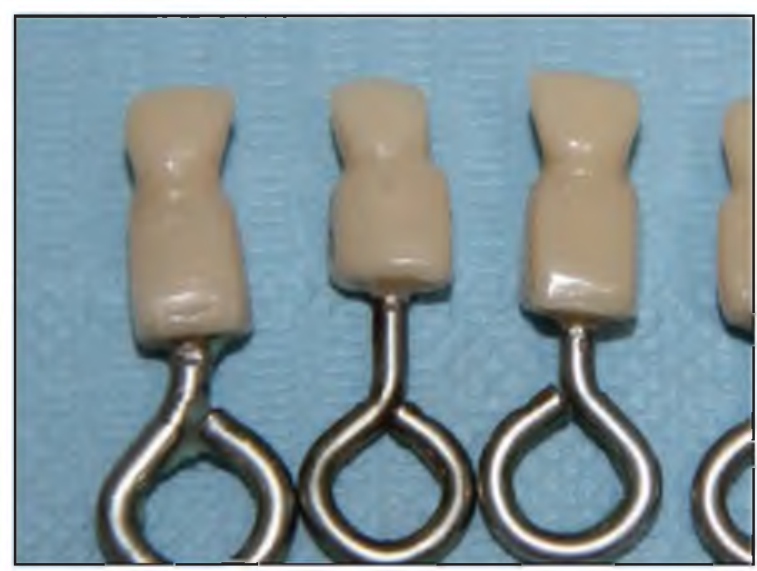

Figura $N^{\circ}$ 6: Stock de dientes de distintos tamaños.

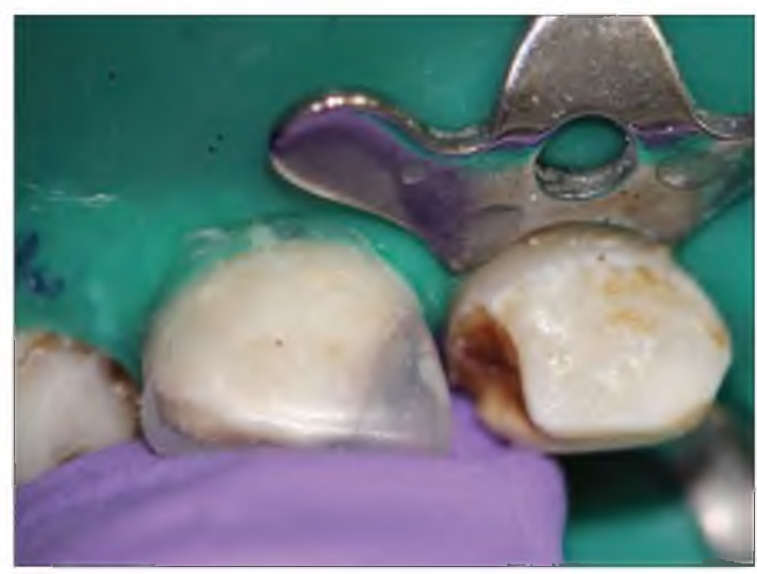

Figura $N^{\circ} 7$ : Prueba de la matriz de acetato. de caries, desde su primera manifestación en la forma de lesión blanca, después la cavitación.

Conociendo aún la permanencia limitada de los dientes deciduos anteriores en la cavidad bucal, presenciamos constantemente la preocupación de los padres por sus hijos $o$, en algunas situaciones, de los mismos niños, con la estética. En la sociedad actual, dientes blancos, bien contorneados $y$ alineados son parte importante del concepto de belleza y salud de los niño (1).

Una diversidad de investigaciones ha sido desarrollada y presentada a la comunidad odontológica con respecto al tratamiento de la lesión de caries a través de técnicas restauradoras. Sin embargo, a pesar de saber que la odontología restauradora no es la "solución" para el problema de la caries dental, se debe tener en cuenta que al presentarse una cavidad, y estando previamente el paciente saneado (adecuación del medio), preparado y educado odontológicamente, se puede contar con una solución basada en el empleo de la técnica restauradora, con el propósito de un restablecimiento anatómico funcional (3).

Cuando Los dientes anteriores están comprometidos por caries dental, inmediatamente se piensa en el factor estético. Sin embargo, no se debe dejar de comentar que antes de la estética existe un ítem más importante: la función (3).

En odontopediatría, la integridad de los dientes y arcadas son de importancia primaria para el mantenimiento de la oclusión, estética, fonética y bienestar psicoemocional del niño. Además, debemos recordar el papel de la dentición temporal en relación al correcto establecimiento de la dentición permanente (2).

No podemos olvidar que la rehabilitación debe estar de acuerdo con las necesidades del niño, englobando los aspectos curativos y restauradores, inserido dentro del contexto de promoción de la salud (4).

Para la rehabilitación coronal, tenemos a las coronas de policarbonato, que son las indicadas cuando hay una excesiva destrucción coronal, sin embargo, estas no se encuentran comúnmente en el mercado. Otras opciones para restaurar dientes anteriores, son restauraciones indirectas con resina, coronas de porcelana y las coronas de acero con carilla para dientes anteriores; pero en ambos casos son muy laboriosas, además requiere mayor colaboración del paciente. Para las restauraciones indirectas con resina, la preparación debe ser expulsiva en toda su extensión, con término cervical en chaflán largo redondeado con ángulos también redondeados (5).

Las coronas realizadas de forma directa son en verdad grandes restauraciones de resina compuesta. Por ser 
realizadas de forma directa no necesitan pasar por procedimientos como impresión y confección de las coronas en el modelo, disminuyendo el número de sesiones (2).

Para ayudar a realizar ésta técnica podemos utilizar matrices transparentes en forma de coronas que facilitan y agilizan este trabajo, además de ofrecer un buen acabado final (2).En el área de odontopediatría, evaluaciones clínicas controladas realizadas en estético. Por otro lado, la satisfacción y aceptación de los padres con el resultado estético, tamaño, forma, y durabilidad ofrecida por coronas metálicas con frente estético en sus niños fue alta (93\%) (6).

En el tratamiento de lesiones extensas en incisivos temporales con composite, mediante la utilización de preformas de acetato transparentes y removibles como matriz, el procedimiento se inicia con la selección del tamaño mesiodistal adecuado de la corona de acetato

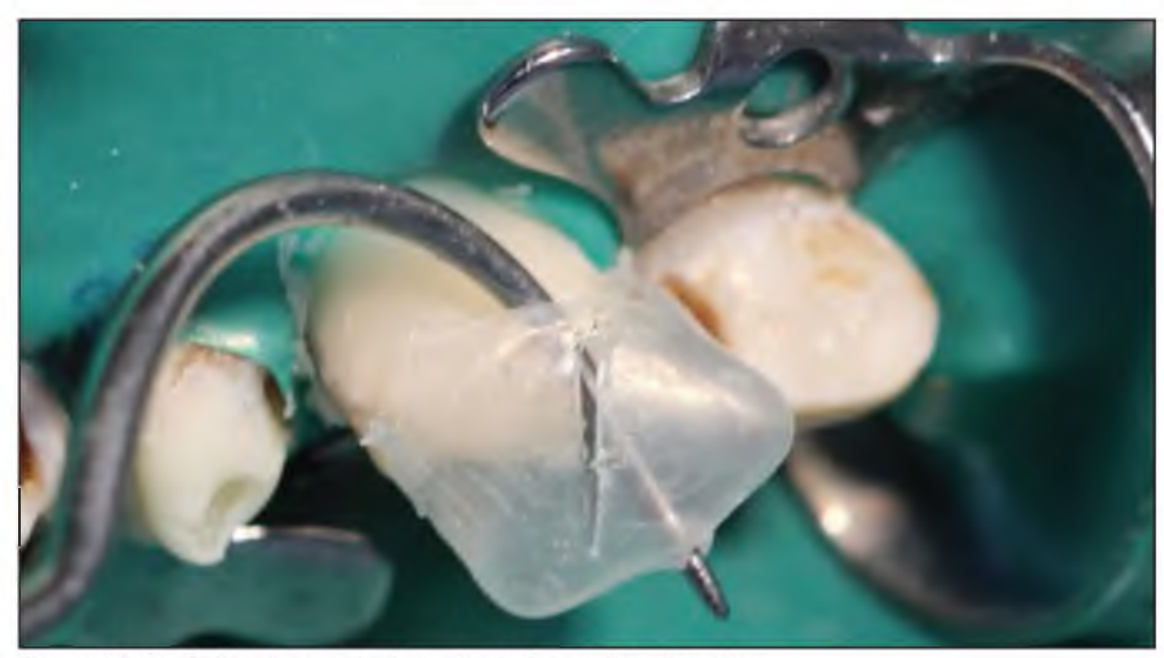

Figura $N^{\circ} 8:$ Retiro de matriz después de fotopolimerización.

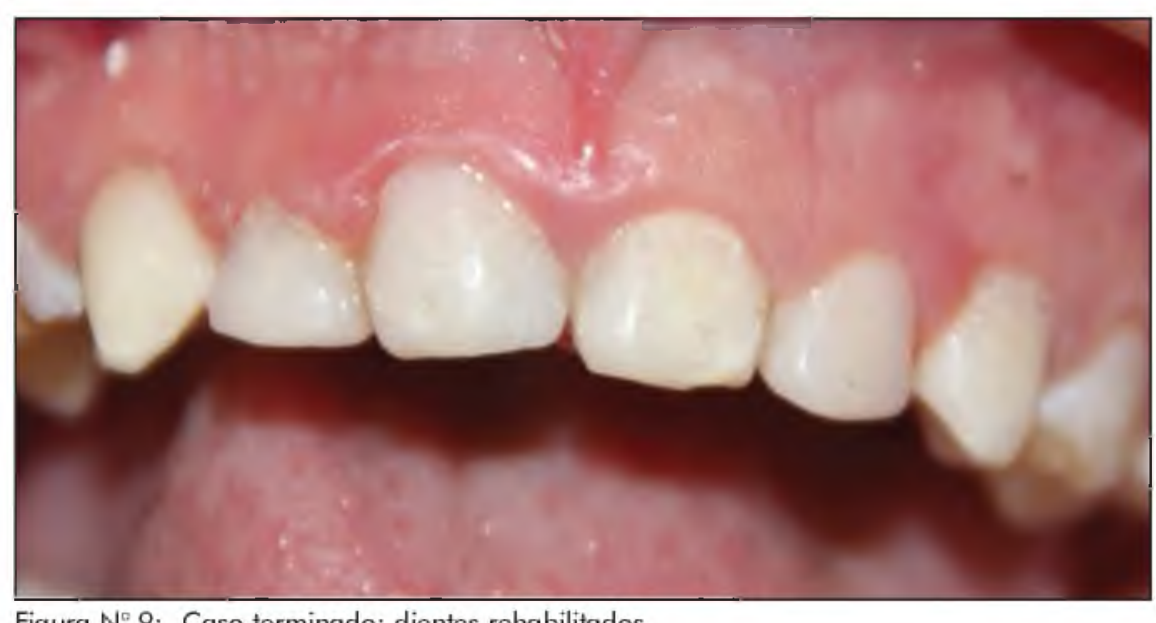

Figura $N^{0}$ 9: Caso terminado: dientes rehabilitados.

dientes primarios anteriores con coronas de veneer o de metal con frente estético (NuSmile) obtuvieron un éxito estético de $91 \%$ y resistencia a fractura de 86 $\%$, mostrando que ese tipo de restauración a pesar de atender la demanda estética del paciente, aún posee limitaciones mecánicas como la fractura, fractura precoz de la porcelana, y despigmentación de su frente estético. Así mismo, otra limitación de ese tipo de coronas fue su sellado marginal por bruñimiento en la región lingual asociado a la fractura del frente y con la selección del color del composite. La corona de celuloide confiere a la restauración una superficie lisa que no requiere pulido. Únicamente cuando se retira el aislamiento y se comprueba la oclusión, si a nivel gingival se detectan rebosamientos, o en las zonas de contacto debe retocarse la oclusión, se procederá al pulido en la forma habitual (7). 


\section{CONCLUSIONES}

Se restituyó la guía anterior de la dentición primaria con las coronas de resina a través de la matriz de acetato, se lograron alinear los dientes según el biotipo facial lo que permitió darle una estética óptima y al tener la dentición primaria completa la caja de resonancia permite una correcta fonación; se devolvió la función de los dientes anteriores, que es la de corte, lo cual permite realizar la primera fase de la masticación; luego según refirió la madre en el primer control realizado después de una semana, se evidenció un cambio positivo notable en cuanto a la autoestima, logrando que el niño sonría abiertamente, dejó de ser objeto de burla, mejoró la interrelación con los compañeros del colegio y sobresale en la actividad académica, lo mismo sucede en el vecindario y la familia muy alegre por ello.

\section{REFERENCIAS BIBLIOGRÁFICAS}

1. Toledo OA. Odontopediatria Fundamentos para a Práctica Clínica. 2ª ed. São Paulo: Premier; 1996.

2. Nahás MS. Odontopediatría en la Primera Infancia. Sã̃o Paulo: Santos; 2009.

3. Guedes-Pinto AC. Rehabilitación Bucal en Odontopediatría. Atención Integral. 1 ed. Colombia: AMOLCA; 2003.

4. Oliveira LB, Tamay TK, Wanderley MT, Rodrigues CR. Planejamento rehabilitador em odontopediatria no contexto de promoção de saúde. Arch. Odont. 2003.

5. Elías PM, Arellano SC. Odontología para bebés. Fundamentos teóricos y prácticos para el clínico. 1@ ed. Madrid: Ripano; 2013.

6. Bönecker M, Abanto J, Nahás MS, et al. Problemas bucales en odontopediatría: Uniendo la evidencia científica a la práctica clínica. Madrid: Ripano; 2014.

7. Boj JR, Catalá M, García-Ballesta $C$, et al. Odontopediatría: La evolución del niño al adulto joven. 1a ed. Madrid: Ripano; 2011.

8. Corréa $N P$, Trindade $C P$ A importancia da reabilitaçāo bucal na recuperaçāo funcional e emocional da criança. São Paulo: Santos; 2002.

9. Braham RL, Morris ME. Odontología Pediátrica Operatoria dental. Buenos Aires: Médica Panamericana; 1984.

10. Baratieri LN. Estética Restaurações adesivas diretas em dentes anteriores fraturados. São Paulo: Santos; 1995.

11. Ferreira LM, Guedes-Pinto AC. Eduçâo do paciente em odontopediatria. São Paulo: Santos; 1997

12. Granath L, Mejáre I, Raadal M. Cárie dentária: Tratamento operatório. $2^{\mathrm{a}}$ ed. São Paulo: Santos; 1995.
13. Cameron A, Widmer R. Manual de odontología pediátrica. Madrid: Harcourt Brace; 1997. 